\title{
Physics from open string wavefunctions
}

\author{
Pablo G. CAMARA* \\ CPHT, Ecole Polytechnique and CNRS, \\ F-91128, Palaiseau, France. \\ E-mail: pablo.camaralcpht.polytechnique.fr

\section{Fernando G. MARCHESANO} \\ PH-TH Division, CERN, \\ CH-1211 Geneva 23, Switzerland \\ E-mail: fernando.marchesano.buznegodcern.ch
}

D-branes provide a simple framework for embedding semi-realistic chiral gauge theories within String Theory. Some of these stringy setups admit a higher dimensional field theory description in terms of open string wavefunctions. These play a role analogous to vertex operators in CFT, allowing to compute $4 \mathrm{~d}$ low energy observables in terms of overlap integrals. In this contribution we briefly review the state of the art of these techniques, applied to D-brane model building.

European Physical Society Europhysics Conference on High Energy Physics

July 16-22, 2009

Krakow, Poland

${ }^{*}$ Speaker. 


\section{Introduction}

Despite the difficulties to accommodate the Standard Model (or extensions thereof) within string theory, it is fair to say that significant progress in this direction has been achieved during the last decade. Such progress has in turn led to estimate that there is a large number of realistic vacua within the string theory landscape, all of them reproducing our current understanding of High Energy Physics. Helas, to date not a single example of such realistic vacuum has been constructed, which leads to wonder about the accuracy of the above estimate.

To some extent, part of the problem is due to the fact that most developments in understanding the string landscape have been performed in the framework of type II string theories with background (closed string) fluxes. While remarkably fruitful, these vacua lack an accurate description in terms of a $2 \mathrm{~d}$ Conformal Field Theory (CFT) describing the propagation of strings in time, which in general jeopardizes the computation of the $4 \mathrm{~d}$ effective theory obtained from them.

Fortunately, the situation is improved for those vacua that can be described at low energies via 10d supergravity. In those cases, higher dimensional geometrical quantities encode the most relevant information about the $4 \mathrm{~d}$ physical observables. In particular, in some setups the spectrum of string states and their interactions can be described in terms of higher dimensional wavefunctions and their overlap integrals, which somehow allows to compensate the lack of CFT techniques.

The aim of this contribution is to review some of the efforts made in the literature to embed the Standard Model in compactifications of type II String Theory and to extract their resulting 4d physics. As in these vacua the Standard Model degrees of freedom arise from open strings ending on D-branes, the latter computation naturally involves understanding the physics of open string wavefunctions.

\section{D-brane model building}

We can distinguish two major (duality related) lines of research in trying to embed the Standard Model within String Theory: heterotic model building and D-brane (or type II) model building ${ }^{1}$. In this contribution we will focus on the latter [1], for whose the Standard Model degrees of freedom necessarily arise from open strings. At low energies open strings lead to $U(N), S O(2 N)$ or $U S p(N)$ gauge theories localized in the worldvolume of $\mathrm{D} p$-branes, whereas closed strings lead to type II supergravity multiplets. In this particular way that the two major features of String Theory, gravity and gauge theories at low energies, are realized in the context of D-brane model building.

There are several constructions within D-brane model building leading to $\mathscr{N}=1$ theories in $4 \mathrm{~d}$. Consider for example the case of $N_{a}$ intersecting D6 ${ }_{a}$-branes wrapping 3-cycles $\Pi_{a}$ on a factorized torus $T^{6}=\left(T^{2}\right)^{1} \times\left(T^{2}\right)^{2} \times\left(T^{2}\right)^{3}$. Each 3-cycle is described in terms of three pairs of integer numbers $\left(n_{a}^{i}, m_{a}^{i}\right)$, which represent the wrapping numbers along the two directions of $\left(T^{2}\right)^{i}$

$$
\Pi_{a}=\left(n_{a}^{1}, m_{a}^{1}\right) \otimes\left(n_{a}^{2}, m_{a}^{2}\right) \otimes\left(n_{a}^{3}, m_{a}^{3}\right)
$$

The spectrum of such construction can be computed by performing an expansion in open string oscillator modes. At the massless level, each stack of D6 ${ }_{a}$-branes will engender an $\mathscr{N}=4 U\left(N_{a}\right)$

\footnotetext{
${ }^{1}$ We could have also added F-theory model building to this list, whose bases are being developed these days.
} 


\begin{tabular}{|c||c|c|c|}
\hline$N_{\alpha}$ & $\left(n_{\alpha}^{1}, m_{\alpha}^{1}\right)$ & $\left(n_{\alpha}^{2}, m_{\alpha}^{2}\right)$ & $\left(n_{\alpha}^{3}, m_{\alpha}^{3}\right)$ \\
\hline \hline$N_{a}=3$ & $(1,0)$ & $(3,1)$ & $(3,-1)$ \\
\hline$N_{b}=1$ & $(0,1)$ & $(1,0)$ & $(0,-1)$ \\
\hline$N_{c}=1$ & $(0,1)$ & $(0,-1)$ & $(1,0)$ \\
\hline$N_{d}=1$ & $(1,0)$ & $(3,1)$ & $(3,-1)$ \\
\hline
\end{tabular}

Table 1: Four stack D6-brane local model leading to the chiral content and gauge group of the MSSM.

Super Yang-Mills (SYM) theory in $4 \mathrm{~d}$. In addition, at the intersection between two stacks of D6 $a_{a^{-}}$ and $\mathrm{D}_{b}$-branes there will be stretched open strings with twisted boundary conditions. Generically, these lead to chiral left-handed fermions transforming in the bifundamental representation $\left(N_{a}, \bar{N}_{b}\right)$ of $U\left(N_{a}\right) \times U\left(N_{b}\right)$ as well as to scalars whose masses depend on the particular angles of the intersection. In the particular case where each stack of D6 ${ }_{a}$-branes satisfies the condition

$$
\sum_{i=1}^{3} \frac{m_{a}^{i}}{n_{a}^{i}} \tau^{i}=\frac{m_{a}^{1} m_{a}^{2} m_{a}^{3}}{n_{a}^{1} n_{a}^{2} n_{a}^{3}} \tau^{1} \tau^{2} \tau^{3}
$$

with $\tau^{i}$ the complex structure moduli of $\left(T^{2}\right)^{i}$, each intersection preserves $\mathscr{N}=1$ supersymmetry and hence its massless content can be arranged into $4 \mathrm{~d} \mathscr{N}=1$ chiral multiplets. In general, the cycles $\Pi_{a}$ and $\Pi_{b}$ will intersect several times, as given by the intersection number

$$
I_{a b}=\prod_{i}\left(n_{a}^{i} m_{b}^{i}-n_{b}^{i} m_{a}^{i}\right)
$$

leading to $I_{a b}$ replicas of the above chiral multiplet.

Global consistency and cancelation of anomalies will in general require also the presence of an orientifold projection. In practice this amounts to introduce special 3-cycles, dubbed O6planes, which are invariant under the orientifold involution $\mathscr{R}$, together with extra D6 $a_{a^{*}}$-branes with wrapping numbers $\left(n_{a^{*}}^{i}, m_{a^{*}}^{i}\right)=\left(n_{a}^{i},-m_{a}^{i}\right)$, that are the image of $\Pi_{a}$ under the action of $\mathscr{R}$.

Open strings in the $a b^{*}$ and $a a^{*}$ intersections lead respectively to $I_{a b^{*}}$ chiral fermions in the $\left(N_{a}, N_{b}\right)$ representation of $U\left(N_{a}\right) \times U\left(N_{b}\right)$ as well as to fermions in symmetric or antisymmetric representations. Moreover, for branes invariant under $\mathscr{R}$ the gauge group is reduced to $S O\left(N_{a}\right)$ or $U S p\left(N_{a}\right)$, depending on their relative orientation with respect to the O6-plane.

The above framework provides then the required building blocks to construct semi-realistic chiral gauge theories consistently embedded into a consistent theory of quantum gravity. One of the most successful local examples is given by the four stack set of D6-branes with wrapping numbers given in Table 1 [2]. At low energies the gauge group is given by a left-right supersymmetric model, $S U(3) \times S U(2)_{L} \times S U(2)_{R} \times U(1)_{B-L}$, which can be spontaneously broken to $S U(3) \times S U(2)_{L} \times$ $U(1)_{Y}$, with precisely the same chiral content than the MSSM.

\section{Magnetized D9-branes and open string wavefunctions}

Intersecting D6-branes are T-dual to configurations of magnetized D9-branes, which not only span the $4 \mathrm{~d}$ non-compact Minkowski directions, but also the whole $6 \mathrm{~d}$ internal space of our string 
theory vacuum. For toroidal setups, the magnetization of the D9-branes is related to the wrapping numbers of the intersecting D6-branes in the original configuration as

$$
F_{z^{k} z^{k}}=\frac{\pi i}{\operatorname{Im} \tau^{k}}\left(\begin{array}{llll}
\frac{m_{a}^{k}}{n_{a}^{k}} \mathbb{I}_{N_{a}} & & \\
& \frac{m_{b}^{k}}{n_{b}^{k}} \mathbb{I}_{N_{b}} & \\
& & \ldots
\end{array}\right)
$$

Roughly speaking, the D-brane magnetization Higgsses the gauge group to $G_{u n b r}=\prod_{\alpha} G_{\alpha}$, giving rise to charged W-bosons, fermions and scalars which transform in bifundamental representations of the gauge group. One can easily see that the resulting spectrum matches the one in the dual configuration with intersecting D6-brane branes. Moreover, the wavefunctions of these charged fields experience localization effects in the internal space, quite in analogy with the localization at the intersections in the dual setup.

Interestingly, at low energies magnetized D9-branes admit a description in terms of 10d field theory, where the D9-brane degrees of freedom are described by the 10d Super Yang-Mills action

$$
S=-\int d x^{10}(\operatorname{det} g)^{1 / 2} \operatorname{Tr}\left[\frac{1}{4} F_{M N} F^{M N}+\bar{\chi} \Gamma^{M} D_{M} \chi\right]
$$

In this sense open string states can be understood as wavefunctions, and more precisely as eigenfunctions of the internal Dirac and Laplace operators

$$
D_{m} D^{m} \Phi^{i}=-m_{\Phi}^{2} \Phi^{i}, \quad \gamma^{m} D_{m} \psi=m_{\psi} \psi^{*}
$$

arising from standard Kaluza-Klein reduction. The solutions describe Landau levels in a magnetized torus, and the number of massless fermionic zero modes is given by the Dirac index $I_{a b}$, which in the dual D6-brane setup corresponds to the intersection number (2.3).

For 2-tori, the solutions to eqs. (3.3) are given in terms of Jacobi $\vartheta$-functions [3]

$$
\psi^{j, I_{a b}}(\tau, z) \sim \exp \left[\frac{i \pi\left|I_{a b}\right| z \operatorname{Im} z}{\operatorname{Im} \tau}\right] \cdot \vartheta\left[\begin{array}{c}
\frac{j}{\left|I_{a b}\right|} \\
0
\end{array}\right]\left(\left|I_{a b}\right| z,\left|I_{a b}\right| \tau\right), \quad j=0 \ldots\left|I_{a b}\right|-1
$$

which transform with the right $U(1)$ charge under global monodromies. Similarly, for higher dimensional factorizable tori, the wavefunctions are given by product of $\vartheta$-functions.

Open string wavefunctions are the target space analogous of vertex operators in the worldsheet CFT. In particular they satisfy an operator product expansion relation, which allows to compute physical observables as overlap integrals of wavefunctions. Thus, for instance, $4 \mathrm{~d}$ Yukawa couplings can be expressed as [3]

$$
Y_{i j k}=\int_{T^{2}} \psi_{i}^{a \dagger} \Gamma^{m} \psi_{j}^{b} \phi_{k, m}^{c} f_{a b c} \sim \vartheta\left[\begin{array}{c}
\frac{i}{\left|I_{a b}\right|}+\frac{j}{\left|I_{c a}\right|}+\frac{k}{\left|I_{b c}\right|} \\
0
\end{array}\right]\left(0, \tau\left|I_{a b} I_{b c} I_{c a}\right|\right)
$$

This is a neat example where the higher dimensional field theory contains much of the relevant information about $4 \mathrm{~d}$ physical observables. Indeed the computation of Yukawa couplings in the dual D6-branes configuration involves complicated CFT computations in the string worldsheet. 


\section{Open string wavefunctions in flux compactifications}

The above simple example illustrates the computational power of open string wavefunctions in cases where a higher dimensional field theory description is valid at low energies. In this sense, the most interesting cases correspond to situations where there is not known $2 \mathrm{~d}$ CFT description, so that open string wavefunctions become the only mean to compute $4 \mathrm{~d}$ physical observables. That is the case of compactifications which involve curved backgrounds or fluxes for closed string fields.

Background closed string fluxes [ 4 ] have become an essential ingredient in model building in the past few years, mainly because of their utility to lift massless scalars, break supersymmetry or implement cosmological models. The best understood setup where they appear is that of warped Calabi-Yau compactifications with magnetized D3/D7-branes, 3-form RR $\left(F_{3}\right)$ and $\operatorname{NSNS}\left(H_{3}\right)$ fluxes. As the equations of motion require $\mathrm{F}_{3}-\mathrm{SH}_{3}$ to be an imaginary self-dual 3-form, constraints arise on the vev of the axiodilaton $S$, the complex structure moduli and the D7-brane positions.

Some of the semi-realistic models of intersecting D6-branes, like the local MSSM construction in Table 1, admit also a perturbative dual description in terms of magnetized D3/D7-branes. Hence, in those models (or in their global completions) it is possible to implement moduli stabilization or soft supersymmetry breaking through the presence of closed string fluxes [5].

Finally, techniques to compute open string wavefunctions in the presence of closed string fluxes have been recently developed in [6]. In terms of magnetized D9-branes, compactifications with closed string fluxes often involve elliptic fibrations (2-tori fibered over a $4 \mathrm{~d}$ base $B_{4}$ ) with RR 3 -form fluxes [7]. The simplest examples of such fibrations are given by twisted tori, where $B_{4}$ is a four-torus and the fibration is specified by a set of structure constants $f_{b c}^{a}$ for the vielbein 1-forms

$$
d e^{a}=\frac{1}{2} f_{b c}^{a} e^{b} \wedge e^{c}
$$

The higher dimensional description field theory description of D9-branes is now given in terms of type I supergravity. There are, in particular, generalized internal Dirac and Laplace operators which account for the effect of the closed string background on the open string fluctuations. Neglecting the warping, ${ }^{2}$ these read respectively for $\mathrm{W}$ bosons, charged matter fermions and scalars

$$
\begin{gathered}
D^{m} D_{m} W^{\alpha \beta}=-m_{W}^{2} W^{\alpha \beta} \quad ; \quad \Gamma_{(4)}\left(\not D+\frac{1}{4} e^{\phi / 2} F_{3}\right) \psi=m_{\psi} \psi^{*} \\
D^{m} D_{m} \Phi^{p, \alpha \beta}-\left[\nabla_{m}, \nabla^{p}\right] \Phi^{m, \alpha \beta}+2 i \Phi^{m, \alpha \beta}\left(F_{m}{ }^{p, \alpha}-F_{m}{ }^{p, \beta}\right)+e^{\phi / 2}\left(D_{m} \Phi^{n, \alpha \beta}\right) F_{n}{ }^{m p}=-m_{\Phi}^{2} \Phi^{p, \alpha \beta}
\end{gathered}
$$

with $D_{m}$ the internal covariant derivative. Solutions to these equations can be found by using the non-Abelian Fourier transform

$$
\psi_{\vec{\omega}}(g)=\sum_{\gamma \in \Gamma} \pi_{\vec{\omega}}(\gamma g) \varphi\left(\vec{s}_{0}\right)
$$

where $\pi_{\vec{\omega}}(\gamma g)$ are the irreducible unitary representations of the $4 \mathrm{~d}$ gauge algebra [9]

$$
\left[D_{m}, D_{n}\right]=-f_{m n}^{p} D_{p}+i F_{m n}^{\alpha} U_{\alpha} \quad ; \quad\left[D_{m}, U_{\alpha}\right]=\left[U_{\alpha}, U_{\beta}\right]=0
$$

$U_{\alpha}$ being the $U(1)$ gauge generators of the D9-branes.

\footnotetext{
${ }^{2}$ For a discussion of the effect of the warping on open string wavefunctions see [8].
} 
In simple models with just an $S^{1}$ fibration, the resulting wavefunctions are similar to the ones encountered in D9-brane compactifications with oblique magnetization and no closed string fluxes ${ }^{3}$

$$
\psi^{\left(j_{1}, j_{2}\right)} \sim e^{i \pi(\mathbf{N} \cdot \vec{z}) \cdot\left(\operatorname{Im} \Omega_{\mathbf{U}}\right)^{-1} \cdot \operatorname{Im} \vec{z}} \vartheta\left[\begin{array}{l}
\vec{j} \\
0
\end{array}\right]\left(\mathbf{N} \cdot \vec{z} ; \mathbf{N} \cdot \Omega_{\mathbf{U}}\right) e^{2 \pi i\left(k_{3} x^{3}+k_{6} x^{6}\right)}
$$

where $\vartheta$ is the Riemann $\vartheta$-function, whose operator product expansion has been worked out recently [10], and $\mathbf{N}$ is a square matrix, whose diagonal entries contain the quanta of magnetic flux and the off-diagonal entries contain the closed string fluxes. The effective complex structure $\Omega_{\mathbf{U}}$ entering in this expression is related to standard complex structure by an orthogonal rotation, whose angle is given by the ratio between the open and closed flux densities. We refer to [6] for details.

As a byproduct, from the point of view of the dual setup with D3/D7-branes, the analysis in terms of open string wavefunctions reveals that D7-brane Kaluza-Klein modes are not affected by closed string fluxes, whereas D7-brane winding modes suffer Landau degeneracies due to the effect of the closed string flux. Mass formulae for these modes can be found in [6].

\section{Conclusions}

D-branes provide a neat framework to embed semi-realistic chiral gauge theories within String Theory. In those cases where a $10 \mathrm{~d}$ field theory limit is a good description at low energies, the open string degrees of freedom are described in terms of wavefunctions. These play a similar role to vertex operators in CFT, allowing to compute $4 \mathrm{D}$ observables in terms of overlap integrals. In particular we have briefly seen how these techniques allow also to compute physical observables in compactifications with closed string fluxes.

\section{References}

[1] For recent reviews see e.g. R. Blumenhagen, B. Körs, D. Lüst and S. Stieberger, Four-dimensional String Compactifications with D-Branes, Orientifolds and Fluxes, Phys. Rept. 445, 1 (2007) [hep-th/0610327]; F. Marchesano, Progress in D-brane model building, Fortsch. Phys. 55, 491 (2007) [hep-th/0702094].

[2] D. Cremades, L. E. Ibanez and F. Marchesano, More about the standard model at intersecting branes, [hep-ph/0212048]; D. Cremades, L. E. Ibanez and F. Marchesano, Yukawa couplings in intersecting D-brane models, JHEP 0307 (2003) 038 [hep-th/0302105].

[3] D. Cremades, L. E. Ibáñez and F. Marchesano, Computing Yukawa couplings from magnetized extra dimensions, JHEP 0405, 079 (2004) [hep-th/0404229].

[4] For reviews see e.g. M. Graña, Flux compactifications in string theory: A comprehensive review, Phys. Rept. 423 (2006) 91 [hep-th/0509003]; M. R. Douglas and S. Kachru, Flux compactification, Rev. Mod. Phys. 79 (2007) 733 [hep-th/0610102].

[5] F. Marchesano and G. Shiu, MSSM vacua from flux compactifications, Phys. Rev. D 71, 011701 (2005) [hep-th/0408059]; Building MSSM flux vacua, JHEP 0411, 041 (2004) [hep-th/0409132].

\footnotetext{
${ }^{3}$ This analogy between open string wavefunctions can be made precise in terms of an open/closed string duality for these backgrounds, similar to the non-Kähler transitions in the heterotic S-dual compactifications.
} 
[6] P. G. Camara and F. Marchesano, Open string wavefunctions in flux compactifications, [arXiv:0906.3033 [hep-th]].

[7] K. Dasgupta, G. Rajesh and S. Sethi, M theory, orientifolds and G-flux, JHEP 9908, 023 (1999) [hep-th/9908088];

[8] F. Marchesano, P. McGuirk and G. Shiu, Open String Wavefunctions in Warped Compactifications, JHEP 0904 (2009) 095 [arXiv:0812.2247 [hep-th]].

[9] N. Kaloper and R. C. Myers, The $O(d d)$ story of massive supergravity, JHEP 9905, 010 (1999) [hep-th/9901045].

[10] I. Antoniadis, A. Kumar and B. Panda, Fermion Wavefunctions in Magnetized branes: Theta identities and Yukawa couplings, [arXiv:0904.0910 [hep-th]]. 